\title{
Retos y Aprendizajes de Institutos de Investigación Transformadora
}

\author{
Mari Jose Aranguren ${ }^{1}$, Susana Franco ${ }^{1}$, Roberto Horta ${ }^{2 *}$, Luis Silveira ${ }^{2}$
}

Resumen: El objetivo de este artículo es reflexionar sobre el papel que desempeñan las instituciones de investigación académica como agentes de cambio en los territorios. Los institutos de investigación que es posible catalogar como de investigación transformadora tienen como objetivo generar nuevos conocimientos diferentes de los centros de investigación tradicionales, porque su finalidad es ayudar al desarrollo de los territorios. En este documento se analizan dos casos de estudio a partir de la experiencia de los cuatro co-autores, que orientan activamente su investigación hacia acciones transformadoras, en dos universidades jesuitas, una en el País Vasco, España, y la otra en Uruguay. Desde este análisis se identifican los cambios que habría que abordar en las universidades para tener un rol más activo y estratégico en el desarrollo territorial.

Palabras clave: Desarrollo territorial; investigación transformadora; institutos de investigación; universidad

\begin{abstract}
The aim of this paper is to reflect on the role our academic institutions play as agents of change in our territories. Transformative research institutes aim to generate new knowledge different from traditional research centres because the objective is helping the development of their territories. The paper analyse two case studies based on the experience of the four co-authors, who actively orient their research towards transformative actions, in two Jesuit universities, one in the Basque Country, Spain, and the other in Uruguay. From this analysis the article identifies the changes that universities that want to have a more active and strategic role in the territorial development.
\end{abstract}

Keywords: Territorial development; transformative research; research institutes; universities

\section{Introducción}

Junto a los pilares tradicionales de la enseñanza y la investigación, a las instituciones académicas se les pide cada vez más que cumplan un rol más estratégico en los procesos de desarrollo económico. Así se habla en términos de «impacto» o "tercera misión" que deben buscar desarrollar las instituciones universitarias. Por otra parte, hay un creciente interés en torno al papel que los diferentes tipos de instituciones académicas pueden desempeñar como catalizadores de cambios dentro de los territorios donde se ubican, contribuyendo de manera proactiva para dar forma a los procesos de desarrollo socioeconómico.

En este trabajo exploramos algunos de los retos a los que las instituciones universitarias se enfrentan al tratar de desempeñar este papel proactivo y convertirse en agentes de cambio dentro de sus respectivos territorios. Con ese objetivo se analizan dos casos particulares de dentro de un contexto específico de desarrollo territorial, en concreto Orkestra, Instituto Vasco de Competitividad (Universidad de Deusto) en España, y el Instituto de Competitividad de la Universidad Católica del Uruguay (IC-UCU).
El documento está estructurado de la siguiente manera. La sección 2, en base a la literatura de referencia, analiza los nuevos roles de las instituciones académicas a los efectos de contribuir al desarrollo económico de los territorios donde se localizan. En la sección 3 se presenta la metodología de análisis utilizada. En la sección 4 se explicita el contexto regional y nacional de los dos casos analizados: el País Vasco en España, junto con el caso de la Universidad de Deusto, y Uruguay y la Universidad Católica de dicho país. El análisis de los dos casos, Orkestra, una iniciativa específica de la Universidad de Deusto que se creó en 2006 con la misión de apoyar y dar forma a la competitividad Vasca, y el Instituto de Competitividad creado en 2007 en el ámbito de la Universidad Católica del Uruguay, es objeto de desarrollo en la sección 5. El análisis está diseñado para descubrir los desafíos críticos que enfrenta una institución académica que pretende desempeñar un papel en la comprensión y facilitar el cambio necesario para mover una sociedad hacia sus objetivos de desarrollo socioeconómico. La sección 6, desarrolla una serie de reflexiones sobre los elementos clave de los institutos de investigación transformadora a partir del análisis de los casos analizados, y en la sección 7 se cierra con una reflexión final que sintetiza los aspectos que caracaterizan a un instituto de investigación en el marco de una universidad que aspira a incidir en el desarrollo del territorio.

(1) Orkestra- Instituto Vasco de Competitividad, España.

(2) Instituto de Competitividad, Universidad Católica del Uruguay.

* Corresponding autor: rhorta@ucu.edu.uy 


\section{2. ¿Cómo va cambiando el rol de las universidades en la sociedad?}

Tradicionalmente se ha reconocido que las universidades tienen dos funciones principales: la educación y la investigación. Sin embargo, hoy en día, a las universidades, y en consecuencia a las personas que trabajan en ellas, se les pide también que contribuyan al desarrollo del territorio en el que se localizan más allá de las dos funciones principales que le eran propias tradicionalmente (Chatterton y Goddard 2000; Etzkowitz et al. 2000; Karlsen et. al. 2012; Aranguren et al. 2015).

Esto ha supuesto también un cambio importante en la evolución del rol de la universidad, que de entenderse fundamentalmente como una institución cuyo principal énfasis estaba en la libertad e independencia de la investigación académica con el objetivo de "generación de conocimiento por su propio bien" a ser una fuente de conocimiento que es un requisito para el crecimiento económico y buen desarrollo (Audretsch 2013).

Este cambio supone el reconocimiento de un rol más estratégico de las universidades en los procesos de desarrollo económico, un rol que demanda fuertemente una ligazón mucho más fuerte de la universidad y el territorio en el que se sitúa. En esta perspectiva las universidades se comprometen en el desarrollo económico territorial, en el que participan activamente y van configurando las redes de aprendizaje e innovación territoriales y alinean sus propias estrategias a las necesidades del territorio (Uyarra 2010).

En esta línea Drabenstot (2008) sugiere que las universidades pueden jugar dos roles respecto al impulso de la competitividad territorial. Por un lado, ayudando a los territorios y a sus gobiernos a hacer un análisis académico de su posición competitiva y de sus ventajas y desventajas; y por otro, haciendo investigación que responda a las necesidades que plantean los retos de la competitividad territorial. Este segundo rol es también la función que reconoce Porter (2006) a las universidades cuando destaca que éstas deben ser "catalizadoras" del desarrollo territorial.

Es precisamente este rol estratégico el que se está pidiendo a las universidades europeas en la actual política de innovación regional europea, la llamada RIS3, o "estrategias de investigación e innovación para la especialización inteligente". Para cumplir con este rol, las universidades deberían participar en el diseño de la RIS3 junto con el resto de los actores regionales, ir casando de forma dinámica sus capacidades de investigación con las prioridades regionales emergentes y mantener una tensión entre la investigación de excelencia académica internacional que, al mismo tiempo, esté orientada a las necesidades específicas regionales. Lo mismo sucede a nivel latinoamericano, en donde cada vez hay demandas más sofisticadas desde los gobiernos, tanto nacionales y/o regionales como de las empresas, hacia las universidades, en cuanto a buscar soluciones conjuntas a problemas específicos.

Esto supone un reto importante tanto para la universidad, habituada a actuar como institución en general separada y distanciada de la realidad en la que se localiza y fuertemente independiente, como para los diferentes actores de desarrollo territorial que no están acostumbrados a trabajar con académicos.

Entender este rol activo y estratégico para la universidad en la configuración de los procesos de desarrollo territorial implica un foco singular en las ciencias sociales marcando un cambio del típico análisis de la tercera misión de la universidad que trate de analizar la transferencia del conocimiento científico-tecnológico entre la academia y la industria. Cuando en el título de este artículo hablamos de institutos de investigación transformadora nos referimos precisamente a aquellos institutos o centros de investigación que pretenden generar un cambio en el entorno en el que se ubican a través de la investigación. Es decir, más allá del objetivo de generación de nuevo conocimiento habitual en cualquier centro de investigación, pretenden que dicho nuevo conocimiento sirva para cambiar el desarrollo de su entorno.

\section{Metodología}

La reflexión y análisis de los dos casos de este artículo se construyen desde la experiencia de los cuatro autores de la misma. Dos de los autores trabajan en Orkestra y los otros dos en el Instituto de Competitividad de Uruguay. Tres de ellos forman parte de la institución desde su creación (entre 9-10 años) y la otra lleva en la institución más de 5 años; todos actuando en las instituciones en un proceso dinámico de orientar la investigación hacia esa acción transformadora.

Por lo tanto, los estudios de casos están construidos desde la praxis, entendida como la relación estrecha entre una forma de interpretar la realidad y la consecuencia práctica que resulta de este entendimiento que lleva a una acción transformadora. Es la síntesis entre la teoría y la acción, en el que continuamente se reflexiona sobre lo que se hace y sobre lo que se piensa.

Estas reflexiones provienen a veces de lo aprendido en el seno de proyectos concretos desarrollados en cada institución y a veces de la gestión y dirección de equipos y de la propia organización (en ambos casos de la interacción continua de la reflexión y la acción), dado que dos de los autores dirigen actualmente cada una de las dos instituciones. Se aplica por lo tanto la metodología del ciclo reflexivo de Andriessen (2004). 


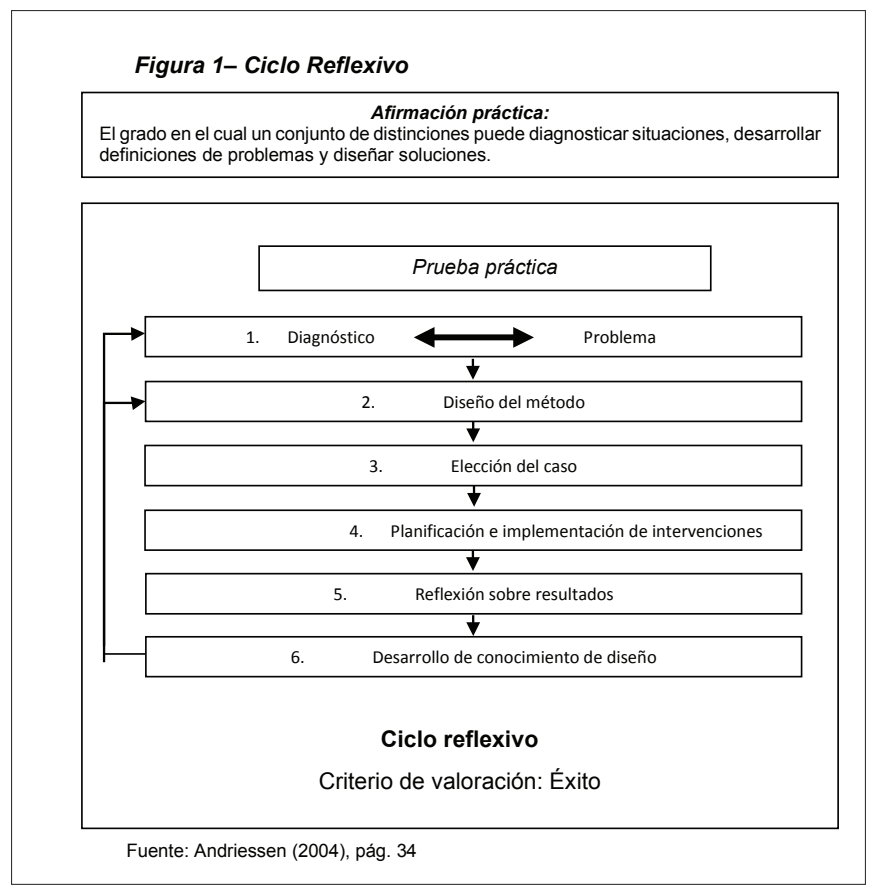

\section{Contexto de los casos de estudio}

Es fundamental considerar la heterogeneidad de los contextos territoriales en los que las universidades deben desempeñar este rol estratégico en los procesos de desarrollo territorial. Por un lado, por los diferentes niveles territoriales en los que las universidades pueden construir sus relaciones (municipales, regionales, nacionales o internacionales) y por otro lado, por los diferentes ámbitos de políticas con los que se construyan estas relaciones (industria, educación, cultura, medioambiente...). Pero sobre los dos elementos anteriores, existe uno más relevante, que se refiere a la tradición o cultura que exista en el territorio sobre la implicación del mundo académico con los agentes socioeconómicos, determinada en parte por las características institucionales del sistema universitario, agencias de gobierno, empresas etc. y por las relaciones potenciales que puedan construirse entre los académicos y agentes territoriales clave. Esta heterogeneidad y especificidad sugiere la importancia de los análisis de caso como metodología apta para explorar los retos a los que se enfrentan las instituciones académicas que busquen tener este rol proactivo en el desarrollo territorial.

\section{1. El Contexto del País Vasco y la Universidad de Deusto}

Situado en la costa norte de España, el País Vasco es una región europea con un importante pasado industrial que durante los últimos treinta años ha seguido un exitoso proceso de transformación que combina crecimiento económico con inclusión social. Es de destacar que el gobierno vasco tiene uno de los niveles más elevados de autonomía en el contexto europeo (Cooke y Morgan 1998) y numerosas competencias en diversas áreas (educación, salud, política industrial y de innovación...).

En ese contexto de autonomía, el País Vasco ha desarrollado una importante red de agentes de ciencia y conocimiento que han contribuido al desarrollo de la región. Desde la década de 1980 se han creado varios centros tecnológicos para apoyar a las empresas y centros de investigación tanto básica como aplicada. A esto se suma el apoyo a las tres universidades vascas, la pública Universidad del País Vasco y las dos privadas (Universidad de Deusto y Universidad de Mondragón). Todo esto configura un complejo sistema de instituciones que apoyan la innovación y la competitividad del territorio que, aunque funciona generalmente bien, también tiene sus debilidades que requieren una racionalización del mismo y una mayor evaluación (OECD 2011; Morgan 2013; Orkestra 2013).

Las universidades en particular presentan varias debilidades. Si bien es cierto que los datos de investigación de las universidades han mejorado en los últimos años (aumentando el número de investigadores, de publicaciones y patentes), ninguna está bien posicionada en los rankings internacionales. Aunque parte de esas debilidades puedan estar relacionadas con una cierta escasez de fondos, hay otros temas, como la falta de autonomía que en general tienen las universidades españolas para determinar políticas que se adecúen al rol que quieran asumir en los territorios donde se ubican. (Orkestra 2013).

Salvo en el caso de la Universidad de Mondragón, que nació ligada al Grupo Cooperativo Mondragón, la tercera misión no ha estado muy incentivada en el País Vasco. En los últimos años, tanto la Universidad del País Vasco como la Universidad de Deusto han buscado avanzar en esta área. La primera uniéndose a varios centros de investigación del País Vasco para crear el proyecto Euskampus y la Universidad de Deusto buscando reforzar su tercera misión a través de la investigación. Como otras universidades jesuitas, la Universidad de Deusto creada en 1886, se enfrenta al reto de cumplir con su misión al servicio de la fe, a través de la ciencia y la cultura. En el año 2004 la universidad creó la Fundación Deusto con el objetivo específico de transferir el conocimiento generado mediante la investigación a la sociedad, con un énfasis particular en actividades de formación e investigación y avance tecnológico, identificando esas necesidades, utilizando los recursos necesarios para llevarlos a cabo y apoyando su promoción y diseminación. En la actualidad la Fundación Deusto consta de tres Unidades de Desarrollo Universitario para realizar estas actividades: Deustotech, Deusto Innovación Social y OrkestraInstituto Vasco de Competitividad.

\subsection{El Contexto de Uruguay y de la Universidad Católica de Uruguay}

Las características del Uruguay de ser un país pequeño y con una diversificación productiva reducida, junto con grandes diferencias de productividad a nivel de sectores, han sido determinantes a lo largo de su historia y han definido que su estrategia de desarrollo no pueda pensarse sin una adecuada inserción al mundo. En las últimas décadas el bienestar promedio de los uruguayos ha mejorado en forma apreciable, ubicando al país en la categoría de "desarrollo humano elevado" según los datos del Índice de Desarrollo Humano 2014 elaborado por el Programa de Naciones Unidas para el Desarrollo (PNUD). El Índice de Progreso Social 2015, por otra parte, posiciona a Uruguay en el lugar 24 a nivel mundial y primero en América Latina. 
Dicha evolución no ha estado ajena a un desarrollo importante de la ciencia, tecnología e innovación en el país. En ese contexto, la estrategia del país ha estado dirigida a "la creación de las condiciones para que el conocimiento y la innovación se vuelvan instrumentos primordiales del desarrollo". Las diferentes políticas públicas han buscado impulsar un desarrollo "intensivo en conocimiento" frente a una estructura productiva que históricamente ha estado basada en la producción de commodities (Camacho y Horta 2014).

En estos procesos el desarrollo de la educación superior en el país ha jugado un rol importante, mostrando un crecimiento apreciable en diversos indicadores, permitiendo afirmar que el crecimiento de la educación superior es una constante que lleva a que el país se ubique dentro de niveles razonablemente competitivos, al menos en lo que se refiere a las instituciones latinoamericanas (CINDA 2011).

A pesar de ello, Uruguay se ha diferenciado del resto de la región en que el sistema universitario privado recién empieza a formalizarse en 1985. Por un siglo y medio, la historia de la educación superior en Uruguay se caracterizó por el monopolio público. La creación de la Universidad Católica del Uruguay (UCU) es la que inicia en 1985 la apertura del sistema universitario, constituyéndose en la primera universidad privada del país.

La UCU se ha definido como una "universidad de docencia con énfasis en la investigación", tendiendo a generar conocimiento relevante para el país y la región. Uno de sus lineamientos estratégicos establecidos en el Plan Estratégico 2012-2017, refiriéndose a la investigación establece: "Incrementar los programas académicos, consolidando líneas prioritarias de investigación, la integración a redes internacionales, el acceso a fondos concursables, así como la visibilidad y comunicación de los resultados".

\section{Los casos de ORKESTRA-Instituto Vasco de Competitividad y del Instituto de Competitividad de Uruguay}

Este apartado se centra en el análisis del Instituto de Competitividad de Uruguay y de Orkestra, que son particularmente relevantes para analizar el rol de las instituciones académicas en el desarrollo territorial por varias razones. En primer lugar, desde su origen se concibieron para cubrir el papel que Porter consideraba que tenían que cumplir las universidades y otros centros de investigación en la promoción del desarrollo territorial. El mismo Michael Porter jugó un papel clave en el diseño de ambos institutos.

\subsection{Orkestra}

Aunque la idea de crear un Centro para la Competitividad del País Vasco se remonta a 1991, cuando se puso en marcha una política de competitividad basada en clústeres, la fundación del Instituto Vasco de Competitividad (que luego pasó a ser conocido como Orkestra), tuvo lugar en abril del 2006.

Desde su fundación Orkestra fue una institución académica integrada en la Universidad de Deusto, pero con una gran autonomía y una estructura de gobierno similar a la de una empresa privada, con un
Consejo de Administración integrado por ejecutivos de firmas que financiaban el Instituto, y un Consejo Asesor integrado por académicos de prestigio internacional.

Gracias a los acuerdos firmados, Orkestra contó con un adecuado financiamiento. A esto se sumaron ingresos captados de fondos de proyectos competitivos que comenzaron a constituir un importante porcentaje de los ingresos a partir del año 2010 y que ahora suponen aproximadamente el 55\% de los mismos.

En cuanto a personal, el Instituto comenzó sus actividades con diez investigadores procedentes de la Universidad de Deusto y tres personas contratadas para las tareas de administración y gestión. Hasta el año 2010 la plantilla se mantuvo bastante estable en torno a 25 personas (15 o 16 investigadores). Entre los años 2010 y 2012 se produjo un periodo de crecimiento hasta aproximarse a las 40 personas (26 investigadores) y actualmente se sitúa en torno a 30 personas (20 investigadores).

Inicialmente se desarrollaron tres áreas de investigación: territorio, innovación y clústeres; emprendimiento; y estrategia empresarial. En 2010 se le añadió una cuarta, energía, con una estructura organizativa diferente. Sin embargo, en el año 2013, como consecuencia de la presencia de culturas organizativas muy distintas en diferentes áreas, y la necesidad de flexibilidad, apertura y generación de liderazgos compartidos para responder a la misión de ser un centro de investigación transformadora, llevaron a iniciar un proceso de cambio organizativo. Este cambio consistió en la eliminación de los departamentos y en el paso a la organización por proyectos y a la generación de espacios de aprendizaje y gestión del conocimiento entre diferentes líneas de conocimiento, entendidas como las capacidades verticales -temas concretos- y las capacidades horizontales, gestión de proyectos y comunicación entre las diferentes personas del instituto.

El trabajo se fundamentaba en tres ejes complementarios, las denominadas tres “i’es": investigación, instrucción e interacción con los principales agentes de la región (gobiernos regional y provinciales, asociaciones clúster, agencias de desarrollo local, empresas, centros tecnológicos y universidades). Un elemento básico de la instrucción lo constituía el curso de Microeconomics of Competitiveness (MOC), que es parte de la red del Harvard Institute of Competitiveness y que la Universidad de Deusto venía impartiendo desde el año 2002, pero que pasó a ser uno de los cursos abanderados del Instituto. La rama de investigación incluía tanto algunos proyectos puramente locales como la participación en redes académicas internacionales y proyectos internacionales con otros centros y universidades. El conocimiento que se va generando se ve plasmado, entre otras formas, en un Informe de Competitividad que se publica cada dos años (Orkestra 2007; 2009; 2011 ; 2013; 2015) y que tiene como objetivo promover la competitividad del País Vasco a través de la investigación de excelencia.

El papel de Orkestra como agente transformador se puede analizar desde una perspectiva tanto cuantitativa como cualitativa. En cuanto a la primera, caben destacar las más de doscientas publicaciones de Orkestra que, en línea con lo establecido por Goddard et al. (2013), han intentado mantener un equilibrio entre una investigación que 
alcance los estándares de calidad y excelencia académicos internacionales y las necesidades prácticas del País Vasco (Karlsen et al. 2012; Aranguren et al. 2012). Se ha participado también en más de doscientas actividades de formación, entre las que destaca el mencionado curso MOC. Este curso ha formado aproximadamente 400 personas de distintos perfiles académicos y profesionales, lo que ha ayudado a construir un lenguaje común de competitividad en la región. A eso se suman las más de trescientas actividades de interacción con diversos agentes nacionales e internacionales.

Con respecto al análisis cualitativo, se ha demostrado (véase, por ejemplo, Aragón et al., 2012; Karlsen et al. 2012; Aranguren et al. 2012) que el trabajo de interacción con los diversos agentes socioeconómicos ha contribuido a reducir los costes de transacción y a generar confianza y, por lo tanto, capital social entre los distintos agentes. Además, ha favorecido la cogeneración de nuevo conocimiento que ha beneficiado tanto a los investigadores de Orkestra, que lo han incorporado a publicaciones académicas, como a los propios agentes. De hecho, la tensión arriba mencionada entre excelencia académica e investigación aplicada a las necesidades del País Vasco, ligada a los efectos de aprendizaje en la práctica de algunas de estas actividades de interacción ha dado lugar a una nueva metodología de investigación, la llamada aproximación de Orkestra a la investigación-acción (Karlsen y Larrea 2015).

La plantilla de Orkestra siempre fue muy consciente de la necesidad de mantener la independencia, frente a los distintos cambios de gobierno que se han producido. Esto ha constituido una prueba de fuego que se ha salvado, en parte, gracias a amistades personales y, en parte, gracias a trabajo intenso entre miembros de la administración y personal de Orkestra, que se reunieron en numerosas ocasiones para construir puentes, desarrollar un lenguaje común y generar visión compartida, con una clara definición de los roles de cada parte.

En resumen, a través de sus actividades complementarias de investigación, instrucción e interacción, Orkestra ha contribuido a la transformación del País Vasco a través de los siguientes mecanismos:

- Contribuyendo a generar un "lenguaje común" sobre competitividad que ayuda a desarrollar una visión compartida de los retos competitivos entre los distintos agentes y a aumentar el capital social de la región.

- Ofreciendo un análisis de la posición competitiva del País Vasco y los retos a los que se enfrenta, que han servido para la toma de decisiones de distintos agentes.

- Ayudando a desarrollar y evaluar las políticas de competitividad de diversas administraciones públicas y convirtiéndose así en una institución de apoyo que sigue manteniendo su independencia.

- Llevando a cabo investigaciones académicas de creciente calidad y relevancia que, al mismo tiempo, contribuyen a difundir el conocimiento sobre el País Vasco por todo el mundo.
A pesar de los logros, Orkestra se enfrenta a varios retos: diversificar y afianzar su financiación, ya que se sigue dependiendo en gran medida de los fondos de los gobiernos regional y provincial y de unas pocas empresas; mantener una relación equilibrada e imparcial con los distintos agentes regionales y sus diversas necesidades; y continuar con su política de internacionalización. Estos retos requieren un replanteamiento constante para centrarse en actividades que permitan mantener ese papel de institución de investigación transformadora, sin caer en los extremos de una investigación de excelencia alejada de la realidad del territorio, ni de actividades de consultoría que impacten en el territorio sin contribuir a generar nuevo conocimiento.

\subsection{El Instituto de Competitividad de la Universidad Católica del Uruguay}

En el ámbito de la Facultad de Ciencias Empresariales (FCE) comenzó a desarrollarse, en el año 2004, el denominado Programa de Competitividad Empresarial y Regional que posibilitó el inicio de una serie de estudios e investigaciones relacionados con la temática de la competitividad y el desarrollo regional y sectorial. Dicho Programa permitió conformar un grupo de académicos que comenzaron a reflexionar sobre la temática de la competitividad, no sólo desde una perspectiva teórica, sino también desde el contacto con la realidad empresarial y territorial.

Dicha reflexión, orientada en seminarios, encuentros y concretada en estudios e investigaciones, demostró que tanto los países como las regiones y las empresas, estaban incorporando en su trabajo diario la búsqueda de estrategias que los diferenciara, valores que los identifique y procesos de construcción competitiva que les permitiera mejorar sus posicionamientos y sus resultados. Se percibió, también, que en diversos países la Academia venía incorporando a sus programas de estudio capacitación específica, investigación y acumulación en la temática de la competitividad, generando centros y/o institutos para colaborar con las empresas y con los gobiernos en los procesos de construcción competitiva. Dichos elementos fueron tenidos en cuenta por la Universidad Católica del Uruguay al crear un Instituto especializado en competitividad, estrategia e innovación, temáticas que complementan, cruzan transversalmente y potencian las acumulaciones especializadas en las áreas de economía y de ciencias empresariales.

En mayo del año 2007 fue creado el Instituto de Competitividad estableciéndose que su principal objetivo fuera el de promover un espacio de excelencia para la generación, acumulación y sistematización de conocimiento en competitividad, estrategia, innovación y emprendimiento, en sus distintos niveles (nacional, regional, sectorial, empresarial), buscando un equilibrio entre la investigación académica y la investigación o consultoría aplicada, priorizando la realización de alianzas nacionales e internacionales.

El Instituto quedó integrado en el ámbito de la Facultad de Ciencias Empresariales, con una estructura organizativa simple y un reducido equipo de investigadores, que se irían ampliando según los proyectos y estudios que se fueran definiendo. Se buscó dotar al Instituto de una 
estructura compuesta por investigadores principales con adecuados antecedentes en los temas de competitividad, innovación y clúster, investigadores junior, asistentes de investigación y secretaría. La dirección del Instituto ha estado a cargo de un Director, designado de entre sus investigadores principales.

Desde el inicio el Instituto fue pensado por parte de sus autoridades, como una organización que debería formalizar, mantener y profundizar redes académicas nacionales e internacionales que posibilitara, con una estructura reducida y un modo de gestión propio, potencializar las bases de conocimiento que le permitieran cumplir la misión prevista en su creación. En ese sentido, se fue desarrollando una amplia red de intercambios académicos con investigadores y centros relacionados con las temáticas de la competitividad y la innovación, a nivel regional e internacional, y formalizando un modelo de operación que partiendo del conocimiento existente en competitividad, clúster e innovación, por un lado, y teniendo en cuenta la propia realidad del país, pudiera generar impactos reales en la competitividad de Uruguay y sus sectores, mediante contribuciones al conocimiento y aportes a la definición de políticas.

En materia de redes de cooperación, se destacó desde un comienzo la participación en la Red del MOC de Harvard Institute of Competitiveness, liderada por el Prof. Michael Porter. La Universidad Católica se había afiliado unos años antes a dicha red con el apoyo de académicos Orkestra. En años sucesivos, el Instituto de Competitividad formalizó acuerdos con otros centros e institutos de investigación en la temática de la competitividad ${ }^{1}$, y se integró como miembro activo a la Red Interamericana de Competitividad que coordina la OEA.

Las líneas de investigación permanentes del Instituto de Competitividad se han orientado en las temáticas vinculadas a la competitividad, la innovación y desarrollo regional y territorial ${ }^{2}$. En el marco de estas líneas de investigación se han realizado diversos proyectos y estudios que han ido generando una determinada acumulación de conocimiento que han permitido ir comprendiendo la situación competitiva de la economía uruguaya y de algunos de sus principales sectores y regiones, a la vez de posibilitar el relacionamiento con el sector público y el sector privado en la contribución de políticas y estrategias tendientes al logro de sus objetivos de un desarrollo competitivo. Dicha acumulación ha significado un aporte desde la academia a una mejor comprensión del desempeño competitivo del país y sus regiones, al difundir un marco conceptual sobre la competitividad nacional y regional, y presentar una evaluación de la situación competitiva y sus desafíos ${ }^{3}$.

\section{Reflexiones y aprendizajes desde las dos experiencias}

En este apartado se presentan las reflexiones sobre los elementos claves de los institutos de investigación transformadora basándonos en el análisis de los dos casos (Orkestra e IC-UCU). Estos elementos, que se describen más abajo, se ilustran en la Figura 2 e incluyen la misión de la organización, su gobernanza y relación con la universidad, una serie de factores internos y las relaciones tanto con agentes locales como con agentes externos.

Figura 2: Elementos clave de los institutos de investigación transformadora

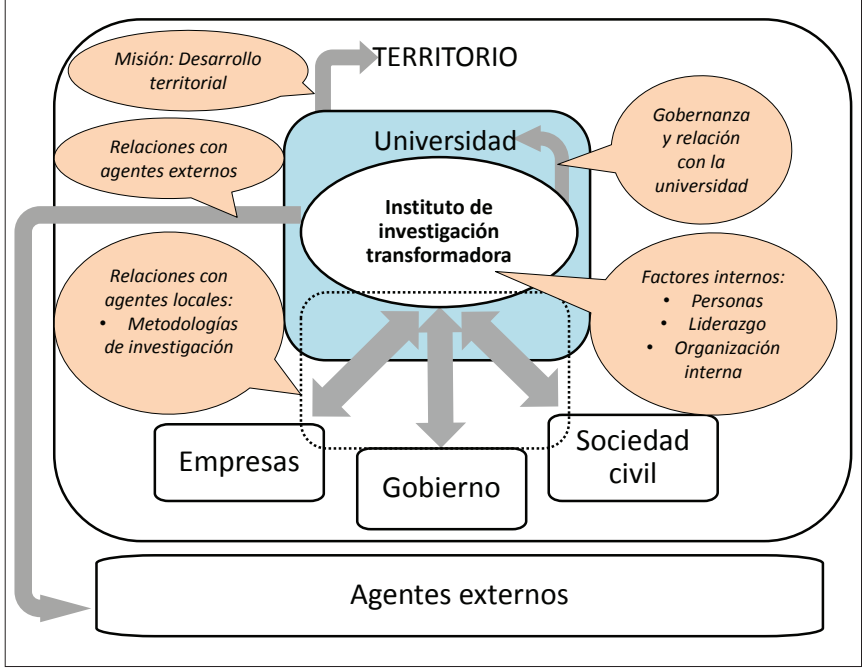

Misión

Tal y como se recoge en Goddard (2000, p. 1), "Aunque las universidades siempre han contribuido al desarrollo social y cultural de territorios en los que se ubican, la agenda de desarrollo regional que está emergiendo requiere un compromiso regional no sólo junto con las actividades de docencia e investigación, sino totalmente integrado con ellos para un reconocimiento formal de la "tercera misión" de la universidad". Esto supone que esa "tercera misión”, el compromiso con el desarrollo regional, se constituye en LA misión de la universidad, que se desarrolla a través de actividades de docencia e investigación. Este rol que se demanda a las universidades para el desarrollo regional en la literatura académica es también uno de los valores fundamentales que la Compañía de Jesús quiere impulsar en sus universidades. Así, Kolvenbach (2000, p. 36) habla de la proyección social de la universidad como una institución transformadora y dice "Toda academia jesuita de aprendizaje superior está llamada a vivir en la realidad social... y a vivir para la realidad social, para aportar la inteligencia universitaria sobre la misma y utilizar la influencia universitaria para transformarla".

Los dos Institutos analizados se crean con la misión de trabajar sobre esa tercera misión de las universidades. Orkestra se crea en el año 2006 en el seno de la Fundación Deusto ${ }^{4}$, con la misión de contribuir a la mejora real de la competitividad del País Vasco y con ello al bienestar de sus ciudadanos y generar a su vez conocimiento de referencia en el ámbito de la competitividad regional. En el caso del IC de la

1 Orkestra-Instituto Vasco de Competitividad, Centro de Competitividad del Maule, Centro de Competitividad Caribe, Sintonía.

2 Véanse por ejemplo los trabajos de Jung et al. (2006), Garbarino et al. (2008, 2014), Jerusalmi et al. (2009) y Horta et al. (2015)

3 Instituto de Competitividad (2014)

4 La Fundación Deusto (CIF G95183828), constituida el 22 de noviembre de 2001, es una fundación sin ánimo de lucro integrada dentro del Sistema Vasco de Ciencia, Tecnología e Innovación. 
UCU, su principal objetivo es el de promover un espacio de excelencia para la generación, acumulación y sistematización de conocimiento en competitividad, estrategia, innovación y emprendimiento, en sus distintos niveles (nacional, regional, sectorial, empresarial), buscando un equilibrio entre la investigación académica y la investigación o consultoría aplicada, priorizando la realización de alianzas nacionales e internacionales. Aunque en el caso de Orkestra está más explícito en su misión el ser un agente de cambio en la mejora de la competitividad del País Vasco, también en el caso del IC de la UCU, en su objetivo principal se explicita la búsqueda de equilibro entre investigación académica e investigación aplicada.

La misión de transformación del territorio a través de la investigación conlleva interacciones intensas con los actores en los procesos de cambio. Esto requiere dedicarle tiempo y, si no sea adquiere el hábito y se le dedica también tiempo a la sistematización y explicitación de nuevo conocimiento (que después ayudan a que los procesos de relación con los propios actores sean más fructíferos por el aprendizaje y cambio que genera el nuevo conocimiento), la misión se ve limitada. Por tanto, la lección aprendida es que la propia misión implica gestionar cuidadosamente el equilibrio entre los procesos de generación de cambio en colaboración con los actores del territorio y la generación, sistematización y explicitación de nuevo conocimiento de relevancia internacional en formato de libros, artículos académicos etc...

\section{Gobernanza y relación con la universidad}

Tal como se recoge en la literatura, el cambio de rol de las universidades hacia una función transformadora del contexto en el que se encuentra requiere la generación de "interfaces de investigación" como centros de investigación universitaria, en el que las unidades básicas como los departamentos se complementan con nuevas unidades y formas de actividad ligadas con el mundo exterior. Como destaca Goddard (2000), estas unidades son responsables de introducir nuevas ideas y promover una cultura más emprendedora en las universidades y tienen así el reto de cambiar las formas de gestión y las estructuras existentes en las universidades.

En el caso de Orkestra, la autonomía de gestión de la que se dotó desde su creación en el 2006 como unidad de desarrollo universitario, con su propio Consejo de Administración (formado por representantes de empresas y entidades públicas patrocinadoras y por miembros nombrados por la universidad) ha sido clave para ir definiendo unas estructuras y formas de gestión diferentes.

Karlsen et al. (2012), al analizar el caso de Orkestra como ejemplo de conexión de la investigación académica con el desarrollo regional en el País Vasco, concluyen que la creación del Instituto, con una clara implicación público-privada, fue central en la transición de procesos de cogeneración de conocimiento experimentales a procesos de cogeneración de forma más sistémica.

Aunque esta autonomía ha sido clave, resulta también muy importante trabajar las sinergias con las facultades y otras unidades de la universidad, para que desde estos centros de investigación se contribuya a la generación de conocimiento en la universidad y se busquen mecanismos para trasladar a actividades de formación universitarias dichos conocimientos. No sólo esto, sino que estos centros podrían tener un efecto de acercamiento de los trabajos de investigación de otros equipos de la universidad a las necesidades del contexto en el que se ubiquen.

En el caso del IC de la UCU, las autoridades universitarias entendieron la necesidad de la existencia de una unidad académica con las características previstas en su reglamento orgánico como "Instituto", es decir, una unidad académica cuya función central sea la investigación en un ámbito o campo epistemológico, donde la unidad está dada por su objeto de investigación. De esta manera se estaba resaltando y priorizando la temática a estudio, ubicándolo en el ámbito de la Facultad de Ciencias Empresariales y, por lo tanto, sujeto a sus definiciones estratégicas. Teniendo en cuenta las principales orientaciones de la UCU y de la FCE, el IC ha tenido autonomía en la fijación de sus planes de investigación y acción, buscando coordinar con las demás áreas de la FCE.

El diferente grado de autonomía desarrollado en el caso de los dos institutos ha venido marcado por su misión. En el caso del IC de la UCU, la unidad ha venido marcada sobre todo por su objeto de investigación, "el qué", y se ubica en la Facultad de Ciencias Empresariales por su adecuado encaje en la misma. En el caso de Orkestra, en la misión se define el ámbito de investigación (competitividad), pero también se destacaba explícitamente por los promotores que se quería que la investigación en competitividad debiera orientarse a generar un cambio en la competitividad real del País Vasco. Esto requería cambiar e innovar en "cómo" se hace investigación en la universidad, para lo que ha sido también importante el cambio en las formas de organización y funcionamiento. Esto conllevaba una mayor autonomía organizativa.

\section{Factores internos: Personas, liderazgo y organización interna}

En cuando a las personas y liderazgo hay varios aprendizajes significativos. El primer aprendizaje importante de los dos casos es que en los centros que busquen impulsar una investigación transformadora el investigador, a diferencia de lo que ocurre en muchos otros centros de investigación, no es un actor que se limita a analizar y entender un tema concreto y hacer propuestas de cambio, sino que entra a formar parte de ese proceso de cambio junto con el actor de competitividad. El investigador no sólo tiene que tener conocimientos técnicos sino también tiene que tener capacidades para facilitar los procesos de cambio. Esto requiere que una de las motivaciones centrales del trabajo de las personas sea el querer generar ese cambio. También es necesario forjar en el equipo de personas valores como compromiso de transformación con el territorio, lealtad institucional en los procesos de investigación implicados, aportación de una visión crítica y flexibilidad para orientar la investigación a los retos de competitividad del territorio. Todo ello permite generar unos espacios de confianza entre los actores de competitividad y el equipo de investigación que son críticos para trabajar con esta perspectiva de proceso. 
Un segundo aprendizaje es lograr una mezcla de personas locales, que conocen muy bien el contexto en el que se ubican para poder trabajar en retos de desarrollo de su contexto, e internacionales, que traen experiencias y conocimientos nuevos en temas de investigación objeto de estudio. Esta combinación de conocimiento local y global genera muy buenas oportunidades de aprendizaje. En el caso de Orkestra, esto se ha desarrollado en parte contratando investigadores internacionales y en parte a través del trabajo en red con otros centros de investigación de referencia. En el caso del IC de la UCU se ha desarrollado más a través del trabajo en red con otros centros que con la contratación de gente internacional.

Un tercer aprendizaje demuestra que cuando se quiere hacer investigación transformadora, el trabajo en interacción con los actores de competitividad requiere conjugar diferentes capacidades (capacidad de análisis, de conceptualización, de facilitación de procesos, de dinamización de equipos....), que es muy difícil que las tenga una única persona. Esto requiere conformar equipos con diferentes capacidades y roles en los proyectos de investigación, constituyéndose en un factor crítico de éxito. Uno de estos roles, que puede diferir del de una investigación más tradicional, es el de comunicación. Aparte de comunicaciones de carácter académico, en congresos, revistas o libros, una investigación transformadora tiene que ser comunicada a los agentes $y$, para ello, es primordial ser capaz de comunicar los resultados y recibir retroalimentación a través de distintos canales.

Otro aprendizaje sobre esta investigación transformadora es sobre el liderazgo. Los procesos de transformación a través de la investigación no los puede liderar una persona, sino que es clave generar liderazgos compartidos en las organizaciones transformadoras. Como destaca Lowney (2003) uno de las cuatro aportaciones clave de los jesuitas al conocimiento sobre liderazgo es que todos somos líderes y dirigimos todo el tiempo.

En cuanto a la organización interna, tal y como destaca Goddard (2000), los centros de investigación que quieren responder a las necesidades regionales tienen el reto de cambiar las formas de gestión y las estructuras existentes en las universidades. En el caso de Orkestra, la estructura inicial de organización interna era una estructura jerárquica con un Director General y Director Académico, cuatro directores de departamentos con sus respectivos equipos y un área de servicios generales. Sin embargo, después de seis años de funcionamiento, tuvo lugar una reflexión interna en la que se detectaron diferentes problemas: cultura distinta en cada unidad, falta de transversalidad en el desarrollo de proyectos y diferentes formas de interactuar con los actores en cada unidad. Esto llevó a un cambio a organización por proyectos y se ha iniciado un proceso de gestión del conocimiento entre las líneas de conocimiento que comprenden las capacidades que tienen las personas del instituto en un ámbito de conocimiento determinado o en un área más transversal. Esto ha permitido una organización más abierta (ha facilitado que el conjunto de personas del instituto interactúen con diferentes actores de competitividad), flexible (los equipos de los proyectos se van configurando en función de las capacidades necesarias para desarrollarlos) y en el que las personas del instituto tienen una actitud más proactiva (que ha tenido un impacto en los proyectos con financiación adicional que se han ido proponiendo y desarrollando por diferentes personas del Instituto). En el caso de la IC de la UCU, al ser una organización bastante más pequeña que la de Orkestra, en general se ha trabajado en base a una organización por proyectos, donde se amplían las capacidades y roles de acuerdo a las necesidades de los mismos. La estructura permanente del Instituto es la que define las estrategias y sostiene las líneas permanentes de investigación.

\section{Relación con los actores locales}

Un primer aprendizaje muestra que cuando la universidad quiere evolucionar a una transformación del entorno, no todas las metodologías de investigación tienen el mismo impacto. En el caso de Orkestra, su misión de incidir en la mejora real de la competitividad requería innovar en cómo se venía investigando habitualmente en la Universidad. Así, una de las innovaciones de Orkestra ha sido la construcción de una nueva aproximación al cómo se investiga (una Investigación transformadora). Una parte de esta investigación transformadora se ha conceptualizado sobre el enfoque pluralista a la Investigación-Acción (Karlsen y Larrea, 2015). La investigación-acción se fundamenta en que el problema sobre el que se investiga es un problema concreto y real que tiene un agente crítico de competitividad (empresa, administración pública, universidad...). Los investigadores desarrollan un proceso de trabajo con el actor que tiene el reto de competitividad; y con la combinación de las capacidades del actor y del investigador, se van buscando soluciones innovadoras al problema. En este proceso el actor aprende para resolver mejor el problema o reto que tenía, y el investigador explicita el nuevo conocimiento generado en formato de artículos, libros..., de forma que pueda ser válido en otros contextos internacionales. Esta es una aproximación a hacer investigación transformadora, pero una de las riquezas del instituto es la diversidad de aproximaciones que hay para hacer esta investigación transformadora. Así, una de los factores diferenciales del instituto está en la combinación singular del qué y el cómo (siendo el qué el universo temático sobre el que se investiga y el cómo las metodologías de investigación abordadas). Un reto que tiene en este momento Orkestra es explicitar otras formas de investigación transformadoras.

En el caso del IC de la UCU, ha existido la preocupación de generar una investigación relevante a la realidad propia del territorio en el cual se relaciona, lo que ha llevado a avanzar hacia una investigación aplicada, buscando la interdisciplinariedad y en contacto con los actores. En forma simultánea se ha ido comprendiendo que la información que se va generando y el conocimiento acumulado que ella posibilita, adquiere mayor relevancia si quien la genera trasmite la necesaria independencia y transparencia, aportando la confianza y la credibilidad, colaborando hacia un direccionamiento que requiere muchas veces del necesario consenso para construcciones competitivas que siempre son de largo alcance en esfuerzo y resultados (Garbarino, 2011).

\section{Relación con los actores globales}

Otro aprendizaje significativo de los dos casos analizados, en cuanto a las relaciones con los actores territoriales y otros investigadores es que, cuando la misión del centro de investigación es la de contribuir a la transformación del territorio en el que se encuentra, los retos a 
los que responde son locales. Sin embargo, el conocimiento necesario para responder a dichos retos es global, por lo que es clave que estos institutos trabajen en colaboración con personas y centros de investigación de referencia en los temas que son críticos para las transformaciones que quieran abordar. Es más, cuando estos institutos en su trabajo de mejora del contexto en el que se encuentran trabajan con actores locales, aprenden y generan un conocimiento que es crítico también en el contexto internacional. Por lo tanto, otra de las singularidades de este tipo de institutos es su combinación local-global.

Cada uno de los dos Institutos analizados constituyen un agente externo para el otro. En este momento, los dos institutos tienen un proyecto conjunto para el impulso de estrategias de especialización inteligente en Uruguay. Orkestra vienen trabajando con el Gobierno Vasco en el desarrollo de la estrategia RIS3 desde el 2013 y ahora el IC-UCU lo está desarrollando con la Dirección Nacional de Industrias. De la experiencia y aprendizaje de ambos institutos de trabajar en proyectos similares con los actores públicos en un ámbito específico y común, genera oportunidades de aprender de las diferencias y similitudes de ambas experiencias.

\section{Reflexión final}

Tal y como destaca la literatura analizada, a las universidades se les pide hoy en día un rol más estratégico en los procesos de desarrollo económico, un rol que demanda una ligazón mucho más fuerte de la universidad y el territorio en el que se sitúa. En esta perspectiva las universidades se comprometen en el desarrollo económico territorial, en la que participan activamente y van configurando las redes de aprendizaje e innovación territoriales y alinean sus propias estrategias a las necesidades del territorio (Uyarra, 2010).

Esto requiere unos cambios en la universidad, cambios sobre las que es fundamental ir aprendiendo de las diferentes experiencias concretas que quieran desempeñar este papel estratégico en el desarrollo del territorio. Cuando en el título de este artículo hablamos de institutos de investigación transformadora nos referimos precisamente a aquellos institutos o centros de investigación que pretenden generar un cambio en el entorno en el que se ubican a través de la investigación, es decir, quieren desempeñar este rol estratégico en la estrategia del territorio. En definitiva, más allá del objetivo de generación de nuevo conocimiento habitual en cualquier centro de investigación, pretenden que dicho nuevo conocimiento sirva para cambiar el desarrollo de su entorno.

En este artículo se presentan los aprendizajes extraídos sobre los cambios necesarios a abordar en la universidad para impulsar este rol más estratégico en su territorio a partir de la experiencia de dos Institutos de Competitividad (IC de la UCU en Uruguay y Orkestra de la Universidad de Deusto en el País Vasco-España). Ambos institutos tienen la misión de tener un impacto a través de la investigación en la competitividad real de los territorios en los que se ubican, por lo que necesitan equilibrar el objetivo de generación de nuevo conocimiento con el de incidir en la estrategia de su territorio.

Como se puede ver en la Figura 3, la misión de incidir en el cambio en el territorio (en su estrategia) requiere cambiar la forma en que los investigadores de los centros se relacionan con los actores, de forma que éstos pasan de ser objetos de estudio a ser compañeros en el desarrollo de la estrategia del territorio, cada uno con un rol diferente. Esto requiere a su vez un modelo organizativo abierto y flexible que promueva que los investigadores interactúen con otros actores, y que los investigadores tengan la motivación de aprender, no sólo para publicar el nuevo conocimiento, sino también para ser agentes de cambio que ayuden en la estrategia del territorio. Todo ello en colaboración también con agentes externos que faciliten las conexiones con el conocimiento global.

Figura 3 Relación entre los elementos clave de los institutos de investigación transformadora

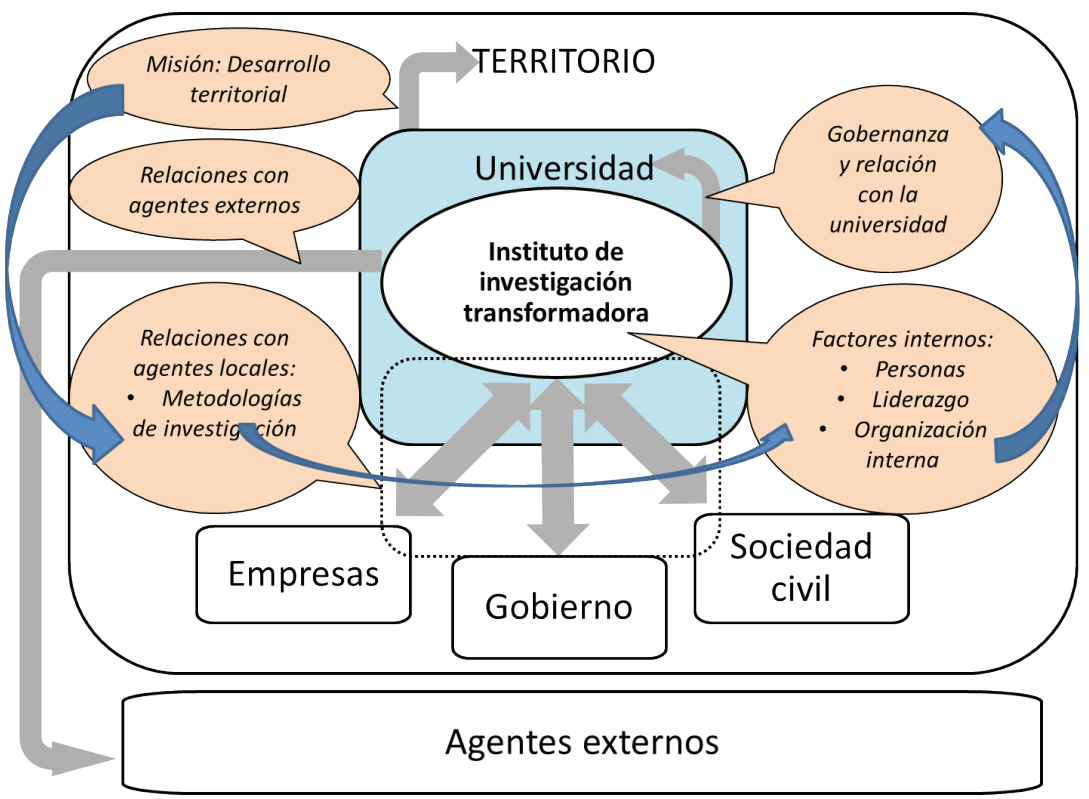

ISSN: 0718-2724. (http://jotmi.org)

Journal of Technology Management \& Innovation @ Universidad Alberto Hurtado, Facultad de Economía y Negocios. 
El desarrollo de estos modelos organizativos abiertos y flexibles se facilita con una modelo de gobernanza que dote de autonomía a los institutos de investigación transformadores, tanto en cuanto a los temas a trabajar como a cómo se aborden los mismos.

Por lo tanto, la misión de un instituto de investigación transformadora requiere nuevas formas de aproximación a la investigación y de interacción con los agentes para lo que contar con investigadores con motivaciones de querer contribuir al desarrollo del territorio a través de la investigación es fundamental. Todo ello se facilita si el centro de investigación tiene autonomía de funcionamiento.

\section{Referencias Bibliográficas}

ANII (2015), ANII Memoria 2006-2013.

Andriessen (2004). Making sense of intelectual capital. Designing a method for valuation of intangibles. Elsevier. UK.

Aranguren, M. J., Karlsen, J., Larrea, M., and Wilson, J. R. (2015). 'The development of action research processes and their impacts on socio-economic development in the Basque Country", en R. Sudgen et al. (eds.) Leadership and Cooperation in Academia: Reflecting on the Roles and Responsibilities of University Faculty and Management, Cheltenham: Edward Elgar.

Aranguren, M. J.; Magro, E.; Navarro, M. y Valdaliso, J. M (2012). Estrategias para la construcción de ventajas competitivas regionals. El caso del Pais Vasco. Marcial Pons. Donosti.

Audretsch, D. (2013)."From the entrepreneurial university to the university for the entrepreneurial society", Jornal of Technology Tranmsfer, 39, 3, pp 313-321.,

Camacho, M. y Horta, R. (2014), El Fomento de la Innovación y el Desarrollo de las PyMES en Uruguay, Red Pymes Mercosur.

Camacho, M., Horta, R. y Rocha, C. (2012), Cluster Analysis Methodology for Developing Economies... Some learned lessons and discussion points. Presentación en Foro Anual del TCI, San Sebastián, País Vasco.

Chatterton, P., Goddard, J. (2000). “The response of higher education institutions to regional needs", European Journal of Education, 35, 4. 475-496.

CINDA (2011). Educación Superior en Iberoamérica Informe 2011, José Joaquín Brunner, editor - coordinador y Rocío Ferrada Hurtado - editora adjunta.

Cooke, P., and Morgan, K. (1998), The Associational Economy. Firms, Regions, and Innovation, Oxford, Oxford University Press.

Drabenstott, M. (2008). 'Universities, Innovation and Regional Development: A View from the United States', Higher Education Management and Policy, 20 (2), 43-55.
Etzkowitz, H., Webster, A., Gebhardt, C., and Cantisano, B.R. (2000). 'The future of the university and the university of the future: evolution of ivory tower to entrepreneurial paradigm, Research Policy, 29, 313-330.

Foray, D. (2013).'The economic fundamentals of smart specialisation', Ekonomiaz, No. 83, pp. 55-82.

Foray, D. and Van Ark, B. (2008). 'Smart specialisation in a truly integrated research area is the key to attracting more R\&D to Europe', in European Commission, Knowledge for Growth: European Issues and Policy Challenges, Brussels: European Commission.

Garbarino, P., Jung, A. y Plottier, C. (2008). La Región desde una Lógica de Clusters: aportes para construcciones estratégicas en Uruguay. ACDE, FKA, Instituto de Competitividad, Departamento de Economía UCU.

Garbarino, P. (2011). "Información, Competitividad y el rol de la academia”, Espectador Negocios, Agosto.

Garbarino, P., Horta, R. y Jung, A. (2014). El Sector Industrial Oleaginoso: Apuntes para un enfoque estratégico. Instituto de Competitividad - UCU.

Goddard, J. (2000). The response of HEIs to regional needs. https://www. google.es $/$ webhp? sourceid=chrome-instant\&ion $=1 \&$ espv=2\&ie $=U T F-$ $8 \# \mathrm{q}=$ goddard + the + response + of + HEIS + to + regional+needs

Goddard, J., Kempton, L, and Vallance, P. (2013). 'Universities and Smart Specialisation: challenges, tensions and opportunities for the innovation strategies of European regions', Ekonomiaz, 83, 83-102.

Instituto de Competitividad (2014). Informe de Competitividad Uruguay 2014, Universidad Católica del Uruguay.

Jerusalmi, C., Camacho, M., Rocha, C. (2009). Cluster Audiovisual en Uruguay, Estudio de caso. Instituto de Competitividad - UCU y PACPYME.

Jung, A., Garbarino, P., Durán, C., Jerusalmi, C. y Plottier, C. (2006). Clusters en Uruguay: Un aporte para el análisis y la discusión de políticas. ACDE, FKA.

Karlsen, J. y Larrea, M. (2015). Desarrollo Territorial e Investigacion Acción. Serie de Desarrollo Territorial. Orkestra y UTN.

Karlsen, J., Larrea, M., Wilson, J. R., and Aranguren, M. J. (2012). 'Bridging the Gap between Academic Research and Regional Development in the Basque Country', European Journal of Education, 47 (1), 122-138.

Kolvenbach, P-H. (2000). The service of faith and the promotion of justice in American Jesuit higher education, San Clara University, 6 de octubre del 2000, http://www.marquette.edu/mission/documents/ TheServiceofFaithandthePromotionofJusticeinAmericanJesuitHigherEducation-Kolvenbach.pdf

Lowney, C. (2003). El liderazgo al estilo de los jesuitas. Grupo editorial Norma. 
Porter, M. E. (2006). 'Colleges and Universities and Regional Economic Development: A Strategic Perspective', Forum for the Future of Higher Education, 2006 Aspen Symposium (http://video.hbs.edu/videotools/play?clip=vc_porter091806, last accessed 8 May 2013).
Uyarra, E. (2010). 'Conceptualizing the Regional Roles of Universities, Implications and Contradictions', European Planning Studies, 18 (8), 1227-1246. 